\title{
Synchrony: A Spiking-based Mechanism for Processing Sensory Stimuli
}

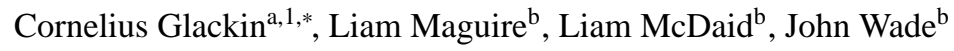 \\ ${ }^{a}$ Adaptive Systems Research Group, School of Computer Science, University of Hertfordshire, College Lane, Hatfield, Hertfordshire, AL10 9AB, \\ United Kingdom. \\ ${ }^{b}$ Intelligent Systems Research Centre, University of Ulster, Magee Campus, Derry BT48 7JL, Northern Ireland.
}

\begin{abstract}
Synchronous behaviour of neurons is both beneficial and detrimental to the neural code. On the one extreme, synchronous firing activity is well known to be a symptom of epileptic seizures, whilst on the other synchrony provides a mechanism for coordinating brain activity. This paper briefly reviews some current thinking with regard to synchrony, and outlines some experiments with LIF neurons that harness near-synchronous states for processing biologicallyrealistic sensory stimuli. Inspired by the topology of neurons in the cochlear nucleus, laterally connected leaky integrate and fire neurons, operating in near-synchronous states, are investigated for their ability to reduce noisy spikes and increase spectral contrast of auditory stimuli. Two connectivity parameters, referred to as connection length and neighbourhood radius, are introduced to configure lateral inhibitory connectivity to generate this neural behaviour. Information-theoretic principles are then employed to quantify the information retained by the coding, and then this is compared to the information retained by the various output topologies.
\end{abstract}

Keywords: Spiking Neural Networks, Synchrony, Lateral Inhibition, Connectivity Regimes, Mutual Information

\section{Introduction}

One of the main aims of neural network research is to understand how neurons respond selectively to certain stimuli, and bind these responses together for joint processing. Synchrony of neural responses, which is characterised by phase-locking of spikes, has long been thought to be the principle means by which variable binding is implemented [1]. The so-called bindingby-synchrony hypothesis suggests that neural circuits integrate distributed circuits into coherent representational patterns. Physical evidence for the hypothesis was found soon after [2], yet it is only relatively recently that networks of spiking neuron models are being experimented with, to study how synchrony works $[3,4,5,6]$. From an experimental viewpoint it is difficult to see how synchrony aids computation. Synchronous states generated in experiments thus far typically show entire populations of neurons firing in phase. If all neurons are firing at the same time, how is synchrony promoting selectivity to certain stimuli?

\footnotetext{
${ }^{*}$ Corresponding author

${ }^{1}$ Email Address: c.glackin2@herts.ac.uk
}

There is still much to understand with regard to synchrony. Nevertheless, there have been some important recent discoveries made. Arguably, it is now widely accepted that it is inhibition not excitation that brings about synchrony, specifically when the rise time of a synapse is longer than the duration of an action potential [3]. It has also been discovered that synchrony arises even with sparse connectivity [4], it can be influenced with modification of inhibitory time constants, and the interplay between these latencies and synaptic coupling strength [5]. Most implementations of synchrony with spiking neurons advocate an all-things-being-equal approach, keeping parameters such as coupling strengths between excitatory and inhibitory cells uniform. Recently, a novel approach advocated modifying the level of coupling with unsupervised learning [6]. Hence, it was reported that a more useful state exists between randomness and full epileptic synchrony that promotes self-organisation of spontaneously active neuronal circuits [6]. It was found that spike timing-dependent plasticity (STDP) decouples neurons (breaks synchrony) by modifying synapses in a non-uniform way. Interestingly, anti-STDP (STDP learning window inverted) brings the neuronal circuit back to a synchronous state. 
Yet it is still difficult to see how this near-synchronous transitory state aids computation.

Since inhibition is crucial for bringing about synchronous states, it is logical to study the biological evidence for inhibitory connectivity, to see if some clues as to how synchrony aids computation can be discerned. In studies of vision, specifically the retina [7], lateral inhibitory connectivity is thought to produce edge/peak enhancement. An analogous process in audition is performed in the anteroventral cochlear nucleus by T-Stellate cells, which extract the profile of the sound spectrum [8]. Yet both studies are unclear as to what specific connectivity regimes produce this computational capability. Therefore this paper advocates the use of specific regimes for lateral inhibitory connectivity. Biologically plausible input data in the form of spoken isolated digits processed using Lyon's Passive Ear cochlear model [9] will be used in the presented experiments. Two connectivity parameters will be used to specify the connectivity regime and demonstrate how specific lateral connectivity can produce edge/peak enhancement, promote feature selection, and reduce noise. The paper builds on previously published work [10] in that it uses information theory to quantify the effects of the various topologies presented.

Within the artificial neural network literature, ideas about information preservation between layers of neurons are well established, arguably since the formulation of Linsker's Infomax Principle [11]. This principle circumvents to classical credit assignment problem of determining which particular synapses within a multiple layer network should be rewarded for correct final output. Instead the infomax principle advocates a local rule of maximum information preservation in each layer, from which emerges a sequence of feature analysing functions. The ehtos behind the infomax principle with regard to preserving the information between the various layers of a network in order to extract features from perceptual data is also relevant for the work presented here. This is achieved by calculating the mutual information between the stimuli and the multiple layers of the various lateral inhibitory topologies presented.

The calculation of mutual information between stimuli and spikes is in general problematic, particularly in the biological setting. For this reason, neuroscientists when trying to determine the information transmission between stimulus and neural response typically use mutual information estimators (for a detailed review of the different estimators see [12]) to cope with the variability of neural response. However, in some scenarios, mutual information calculated directly can be accurate if neural responses are limited to a small set and stable recordings can be made [13]. The stimulus encoding and spiking neuron models employed in this work are deterministic, the data is also tonotopically arranged by frequency, hence a naive mutual information calculation can be made to assess the information preservation within the tonotopic frequency bands of the data. The aim is to evaluate the mutual information retained within these frequency bands and transmitted throughout the various neurons in each layer (which are also tonotopically arranged).

Section 2 outlines the pre-processing required to obtain biologically realistic speech signals. Section 3 demonstrates how the analogue speech signals are converted to digital spike trains. Section 4 provides background on the information theoretic concepts used to analyse the encoing and the various topologies presented. Section 5 outlines how lateral inhibitory networks can be designed to promote feature selection, produce edge/peak enhancement and reduce noise. A short discussion follows this in Section 6, before conclusions are presented in Section 7.

\section{Speech Pre-processing}

The speech samples used are isolated digits from the TI46 database [14]. A sample of digits spoken by 5 female speakers, with 10 utterances for each digit, and 10 total digits ( 0 to 9 ) making 500 speech samples, is selected for this work. Pre-processing of speech information in order to enhance speech features and any resulting recognition is an important undertaking. With regards to the way in which the pre-processing is conducted, there are two main approaches; computational and biologically-inspired respectively. It has long been recognised that sounds are best decribed in the frequency domain. Thus the computational approach begins by performing spectral analysis to decompose the speech sample into its frequency components. Typically, the short-time Fourier Transform is preferred over the standard fast Fourier Transform as it is more suited to time-varying signals. The frequency components are usually distributed along the $\mathrm{Mel}$ scale, which is linear for low frequencies and logarithmic for high frequencies, corresponding to the physical properties of the human ear. Thus the human auditory critical bands are approximated using triangular filters, distributed in a combination of linear and $\log$ positions (there are several methods), and a discrete cosine transform is used to decorrelate the features and produce Mel-Frequency Cepstrum Coefficients (MFCC) [15]. 
The biologically-inspired approach typically involves the use of a cochlear model to extract biologically realistic frequency information from the speech sample. Both methodologies can result in the generation of a frequency-based representation of the sound. For the computational approach this representation is referred to as a spectrogram, and for the biologically-inspired approach a cochleagram. In this work the biological approach is preferred since the increased contrast of the cochleagram over the spectrogram facilitates the encoding procedure [10]. Figure 1 shows a cochleagram generated using Lyon's Passive Ear (LPE) cochlea model [9] for speaker 1, utterance 1, digit 1 (s1-u1-d1) from the TI46 speech corpus [14]. This particular sample was chosen arbitrarily and will be used throughout this paper to illustrate the feature extraction and information theoretic analysis. The cochlea model is employed to approximate the frequency composition along the basilar membrane. The LPE cochlea model contains a notch filter bank which models the sensitivity of the cochlea to certain frequencies. The cochleagram shown in Figure 1 was generated using Slaney's Auditory toolbox [16] with a sample rate of $12.5 \mathrm{kHz}$.

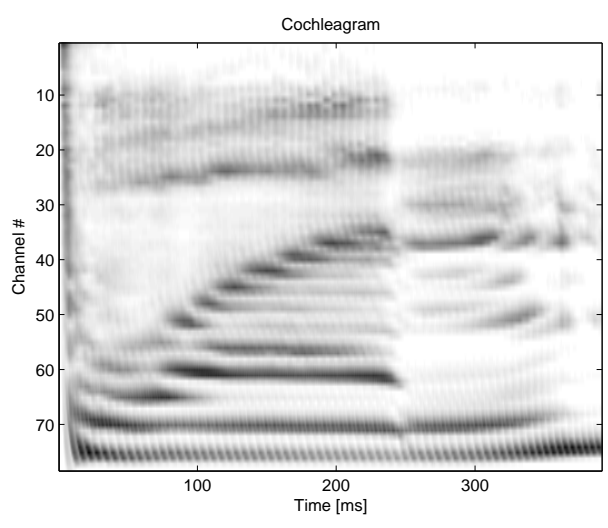

Figure 1: Cochleagram generated using Slaney's Auditory Toolbox [16] for sample: s1-u1-d1

The typical MFCC methodology suffers from the limitation that the temporal nature of the speech sample is treated in an arbitrary way with the features of the sound signal effectively averaged within each frame. Such treatment results in a fixed number of features that are ordered at arbitrary time intervals rather than attempting to preserve the temporal information. This treatment explains why the MFCC technique has been researched extensively in an attempt to improve its performance, particularly with noisy data.

\section{Spike Encoding}

The next step is to obtain biologically realistic spike trains, and this requires a conversion from the continuous data in the cochleagram into discrete spike trains. There are various ways in which this can be performed [19]. The most efficient method considers spike trains as digital signals, since it is thought that it is only the timing of the individual neurons that biological neurons use to communicate with one another. Therefore, the generation of spike trains from the cochleagram may be considered as an analogue to digital conversion. There are algorithms in the literature that have been developed to convert continuous data into discrete spike timing, these are referred to as spiker algorithms $[18,19]$. The two main methodologies are referred to as HSA (Hough Spiker Algorithm [18]) and BSA (Ben's Spiker Algorithm [19]) respectively. Both algorithms utilise a convolution/deconvolution filter that was optimised for encoding/decoding [18] using a genetic algorithm to minimise the error in the encoding and decoding process. For filter specifications, impulse responses, frequency spectra and pseudo code refer to [19]. Figure 2 shows the resulting spike trains generated using BSA.

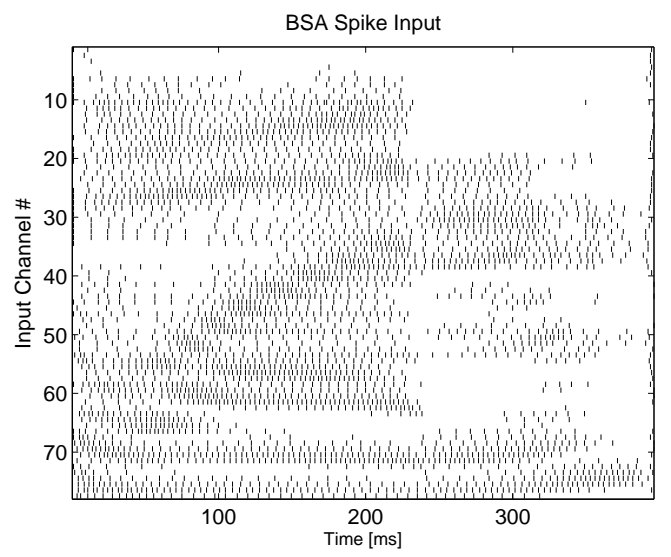

Figure 2: BSA [19] speech encoded spike input for sample: s1-u1-d1

Figure 2 illustrates how the 78 frequency channels generated using the convolution filter and BSA are converted into spike trains. It can be seen from the diagram that individual spike trains begin firing, end firing, and fire maximally at specific times. The literature describes these features of sound signals as onsets, offsets and peak firing rates respectively [20]. Onsets can be extracted [21, 22] from spike trains using spiking neurons with depressing synapses that are configured to consume their synaptic resources when firing in response to the first presynaptic spike in a spike train and 
consuming all their synaptic resources. Offset detection is a little more complicated but can be performed using three neurons, two input neurons connecting to an output neuron. One input neuron is inhibitory and the other input neuron is excitatory with a small delay, for further details see [22].

\section{Analysing Spiking Topologies with Information Theory}

The various raster plot presented throughout this work give a clear pictorial representation of the accuracy of the encoding of the input spike trains (when compared to the cochleagram), and also of the relationship between the spike inputs and the various spike outputs. Whilst this pictorial representation is informative, this work extends on the previous work [10] by attempting to quantify the information each of these figures contains. Information theory provides a vehicle for measuring the information at each stage of the spiking network topologies from the encoding to the output. In particular it would be beneficial to characterise the $m u$ tual information between the cochleagram and the spike input encoding, and the cochleagram and the various spike outputs. The mutual information $I$ between two random variables $X$ and $Y$ is give by [23]:

$$
I(X ; Y)=\sum_{x \in X} \sum_{y \in Y} p(x, y) \log \frac{p(x, y)}{p(x) p(y)}
$$

where $p(x, y)$ is the joint distribution, $p(x)$ and $p(y)$ are the marginals. By calculating the mutual information between cochleagram and input, and cochleagram and output, the amount of information retained by the encoding and the amount of information transmitted by the lateral inhibitory networks can be quantified.

\subsection{Mutual Information between Cochleagram and Spike Encoding}

Armed with the mutual information equation, it is now possible to determine in information theoretic terms the accuracy of the spike encoding performed by BSA [19], this will serve as a baseline for mutual information calculations between the cochleagram and the various output layers of the network. In order to calculate the mutual information $I$ in Equation 1, it is first necessary to calculate the joint distribution $p(x, y)$. Here the random variables $X$ and $Y$ are taken to represent the continuous valued data in the cochleagram and the discrete binary data in the spike encoded input respectively. But herein lies the first difficulty, that of calculating the joint distribution between continuous and discrete random variables.

The approach advocated in this work is to apply the well-known technique of binning to discretise the continuous-valued cochleagram data $X$. Binning the data can be problematic, if done incorrectly it can introduce bias into the mutual information calculation that follows [12]. The idea is to bin the continuous data in a way that maximises the mutual information calculation. It can be shown that maximising mutual information is equivalent to maximising the entropy of the bin probability distribution [23]. It is well known, that the entropy of a particular random variable is maximised when the random variable in question is uniformly distributed [23]. In this way, by ensuring that the number of data points within each bin (for the continuous cochleagram data) are equal. This can be achieved by ordering each continuous value in the distribution of $X$ in order of size and then determining the range of the first $1 / K$ of the total values (where $K$ is the number of bins), and using the range values as limits for the values that are to be placed in the bin. The range of the next $1 / K$ values define the second bin, and this continues until the limits for all the arbitrarily chosen number of bins are defined. In this way each bin encompasses the same number of continuous values (maximising the entropy of the bin distribution), a technique known as adaptive binning [24] or sampling equalisation [25].

The binning of the spike data is less problematic than the maximum entropy binning of the cochleagram data as the spike data is discrete. All that needs to be determined is the binary word length (spike train segment) to be used in conjunction with the continuous cochleagram samples when calculating the joint distribution between the cochleagram data and the spike encoding. The simplest spike binning is to consider word lengths of one bit i.e. two bins, namely 0 or 1 , however this size of bin for the spike data results in low estimations of mutual information. Larger word lengths can be used but for very large word lengths, there is a risk for a given amount of data that the joint distribution will be too sparsely populated. It was determined that the optimum word length for the given amount of data in the speech sample was 3 bits (resulting in $2^{3}$ bins). Even with such a small word length, care needs to taken in the contruction of the joint distribution to determine where continuous data at time $t$ in the cochleagram maps to in the spike encoding. Since the spike encoding employs a convex convolution function which is applied over a time interval of $20 \mathrm{~ms}$, it is necessary to consider that mutual information may be maximised at any point up to $20 \mathrm{~ms}$ before the generation of each spike in the encoding. By implementing 
a time-shift parameter and iterating the construction of the joint and marginal distributions and hence the calculation of the mutual information using Equation 1, it can be determined how long it takes information from the cochleagram to manifest itself in the spike encoding, as is illustrated with Figure 3.

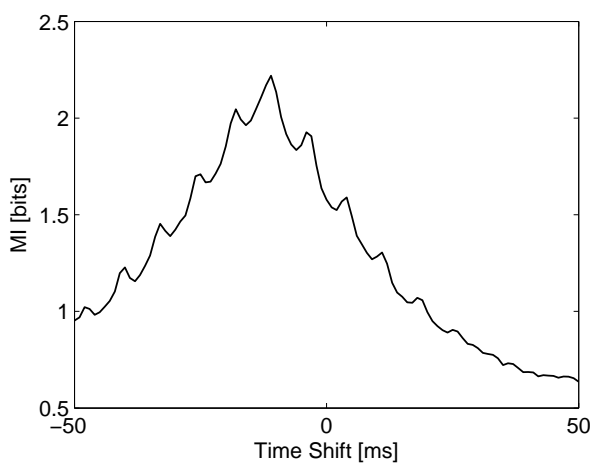

Figure 3: Mutual Information for various time shifts for sample: s1u1-d1

Figure 3 shows the mutual information plotted against a range of negative to positive time shifts. As can be seen from the figure, the mutual information clearly peaks for a time-shift of $-11 \mathrm{~ms}(11 \mathrm{~ms}$ before the generation of the spike at time $t$ ). This coincides with the peak of the convex convolution function employed by the spiker algorithm [19]. BSA encoding uses this convolution function (which was optimised using a genetic algorithm [18]), to smoothly quantise the continuous data to discrete spiking output. The maximum mutual information for the s1-u1-d1 sample is 2.22 bits, meaning that each time step of the continous cochleagram input, 2.22 bits of information is transmitted to the encoded spike output. The mutual information will be calculated throughout the following sections of the paper in order to quantify the effects of the various lateral topologies presented. The next section provides background information about the construction of these topologies.

\section{Lateral Inhibitory Networks}

One way to discover when neurons are firing at the same rate is by synchrony [26]. This can be investigated with a simple experiment originally proposed by Abbott [27]. Figure 4 shows the interaction between two LIF neurons with lateral inhibitory connections, connecting to an excitatory output neuron. Each neuron in the input layer receives as input the inhibitory output of the other. Each of these neurons has its own excitatory input, one receives a fixed firing rate of $25 \mathrm{~Hz}$, the other a firing rate linearly changing from 28.5 to $21.5 \mathrm{~Hz}$.
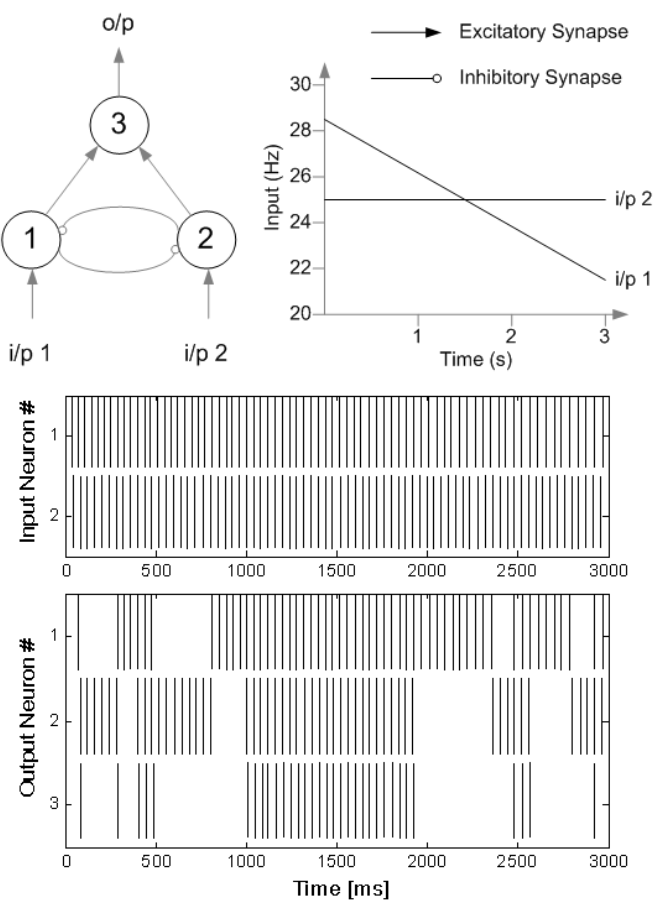

Figure 4: A simple three neuron SNN (top left) used to test the ability of laterally connected neurons to produce coincidental firing and synchrony [27]. The SNN receives two input spike trains, one with a firing rate reducing from 28.5 to $21.5 \mathrm{~Hz}$, the other with a constant firing rate of $25 \mathrm{~Hz}$ (top right). The bottom two subplots show the actual input and output spike rasters of inputs 1 and 2 (i/p 1 and i/p 2) and resultant spike output for neurons 1,2 , and 3 respectively.

As can be seen from Figure 4, when the two input neurons are firing at different frequencies, the input neurons take turns at suppressing the output of one another, depending on spike timing. The output neuron, neuron 3, fires maximally (coincidently) when the two input neurons are firing at the same frequency [27]. Abbott's experiment [27] illustrates how frequency information can be extracted from a spike train. It demonstrates how to find when a spike train is firing at a particular frequency. This experiment is particularly useful from a feature extraction point of view as it can be used to determine when spike trains in the spike array of Figure 2 are firing at similar frequencies. In fact there is no limit to the number of spike trains that can be compared in this way, coincidence detection can be performed for any number of spike trains as long as they have 'sufficient connectivity' [4]. 


\subsection{Connection Length}

The output from the LPE and subsequent BSA encoding of spike trains is tonotopically arranged. Therefore, it does not necessarily make sense to associate every input neuron and hence sound frequency with every other, as this disregards this tonotopic arrangement. It seems more likely that the lateral connectivity of the input layer can be described in terms of a connection length parameter. A particular connection length of $c$ would mean that each input layer neuron is laterally connected to $c$ neurons either side of it. Figure 5 illustrates this idea, the black lines of various styles represent connection lengths between 1 and 3 for an example layer of laterally connected neurons.

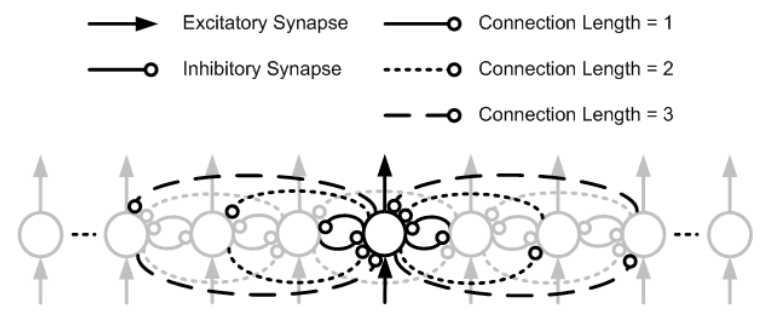

Figure 5: Neurons connected laterally as determined by a connection length parameter

In this way, a layer of $N$ neurons can have a maximum connection length of $c_{\max }=N-1$. In the case of the spike trains in Figure 4, a layer containing $N=78$ neurons similar to those in Figure 2 was implemented with an initial connection length of 0 (i.e. no lateral connectivity). Successive simulations were conducted with the connection length increased by 1 up to the maximum connectivity of $c_{\max }=77$. Figure 6 presents a selection of the spike outputs from these experiments.

As can be seen from Figure 6, synchrony appears with much less than full connectivity, in fact it can be seen to begin to form with neighbourhoods laterally connected to tonotopically adjacent neurons with a small connection length $(c=15)$. Thus, even with sparse lateral inhibition, the layer of neurons in Figure 5 is shown to be capable of attaining a synchronous state in accordance with [4].

\subsection{Multiple Layers of Synchrony}

The layer of neurons with full lateral connectivity produced synchronised spike activity from tonotopically arranged sound information. An obvious line of enquiry is to investigate what happens when the synchronous spike activity is passed through multiple layers of fully connected lateral inhibitory neurons. Figure
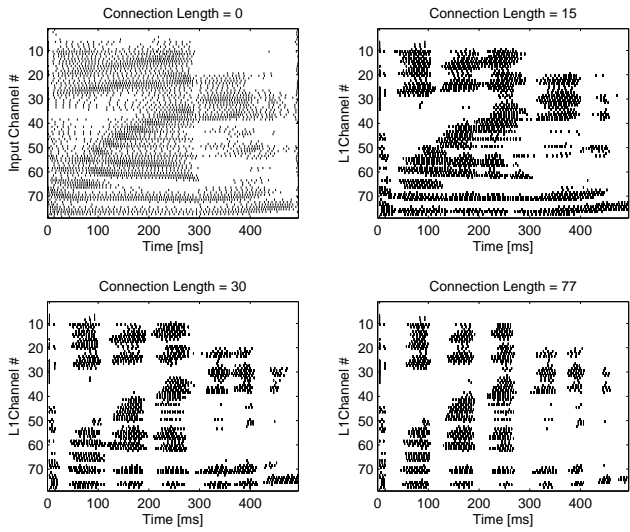

Figure 6: Effects of increasing connection length on synchrony for sample: s1-u1-d1. A sample of results are shown for connections lengths of $0,15,30$, and 77 .

7 shows the input and output of 3 layers of such connectivity.
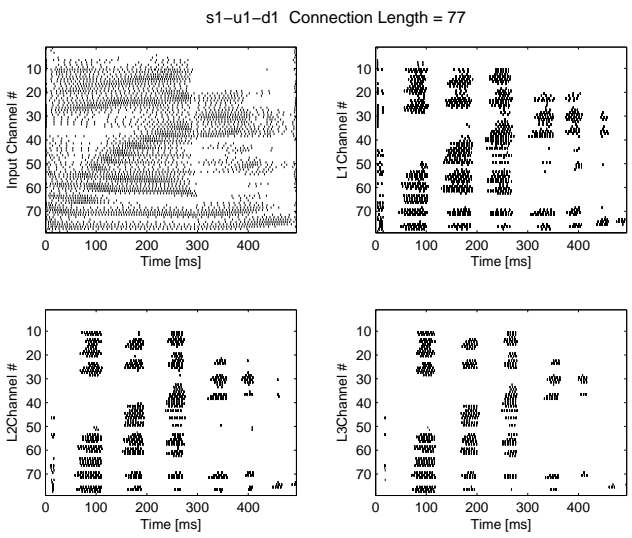

Figure 7: Input spike trains for sample: s1-u1-d1 (top left) routed through three successive layers (L1, L2, and L3) of neurons laterally connected with inhibition.

Figure 7 demonstrates the effects layers of synchrony have on spike input. The output from the first layer is as expected from Figure 6. However, it can be seen that iterating the lateral processing over several layers serves to successively remove more spikes at each layer. The spikes that remain are densely packed by necessity in order to survive this process. Contrastingly, areas of spikes which are not densely packed are removed. The removal of noisy spikes is a feature of this connectivity that is potentially useful, particularly if it can be implemented without the periodic removal of all spikes at regular time intervals in the output, as this represents a po- 
tentially crucial loss of information. Therefore the focus of the remainder of the paper will be to investigate how edges can be enhanced, and how noisy spikes can be removed, but without the periodic removal of spikes associated with such epileptic synchrony. Figure 8 shows the mutual information calculated for the same multiple time shifts and spike word lengths as was used for the encoding. There has been some loss of information compared with the mutual information for the spike encoding, this is to be expected, however later the mutual information across all layers will be discussed to determine how well this topology preserves information in successive layers.

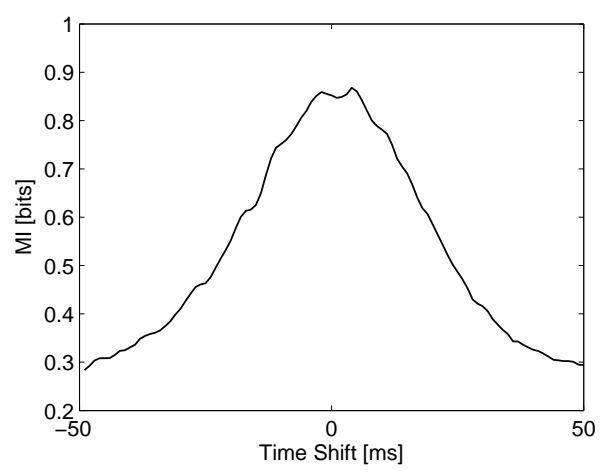

Figure 8: Mutual information between cochleagram and layer 1 synchronous output with multiple time shifts for sample: s1-u1-d1

The mutual information for layer 1 peaks at 0.87 bits, around a time shift of $0 \mathrm{~ms}$. This is precisely why multiple time shifts are examined, for layer 1 there is a 10 $\mathrm{ms}$ delay in inhibitory synapses, hence the mutual information peaks $10 \mathrm{~ms}$ after the mutual information in the encoding.

\subsection{Neighbourhood Radius}

The concept of a neighbourhood in the connectivity of neurons was introduced by Kohonen with the 'winner-take-all' competetive learning algorithm. Essentially, 'winning' neurons had their weights increased along with neurons topologically close to them, i.e. in the same neighbourhood. The idea of defining neighbourhoods can also be introduced in the case of spiking neural networks. In this paper, as already discussed, the connection length parameter defines how tonotopically far away a neuron in the same layer can be connected using lateral inhibition. Another parameter referred to as neighbourhood radius can also be introduced, that describes how neurons that are tonotopically close to one another are not connected laterally. Figure 9 illustrates this idea.

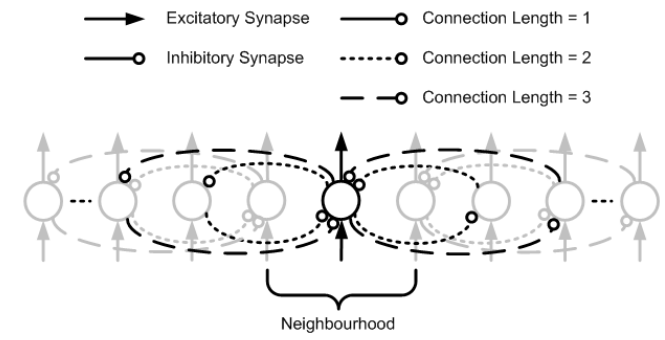

Figure 9: Introducing the neighbourhood radius parameter to layers of lateral connectivity specified by the connection length parameter

In general three distinct feature extraction properties have been observed with different connectivity parameters for randomly selected samples from the TI46 dataset, these are synchrony, edge enhancement, and noise reduction. Suppose one wishes to identify the most important frequency channels for a particular sample, and how the importance of these channels varies over time. In terms of the spike encoded speech sample this means the aim is to find the highest frequency spike trains at each time interval throughout the sample. This can be achieved for a maximum connection length and a relatively smaller neighbourhood size, as illustrated by Figure 10.
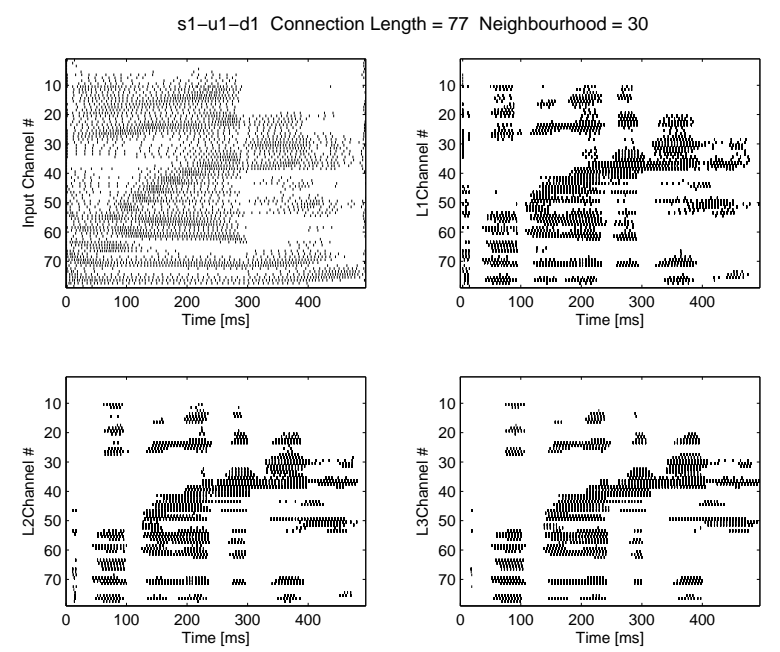

Figure 10: Maximum lateral inhibitory connectivity (connection length $=77$ ) with a neighbourhood radius of 30 for sample: s1-u1-d1. Partial synchrony has removed some of the spikes from the resultant rasters.

\subsection{Edge Enhancement and Noise Reduction}

In order to make meaningful the tonotopic arrangement of the spike encoded sound channels, it follows 
that neurons that are tonotopically distant from one another should have little connectivity. In terms of the connectivity parameters already defined, a less than maximum connection length has interesting effects on small neighbourhoods as is illustrated by Figure 11.
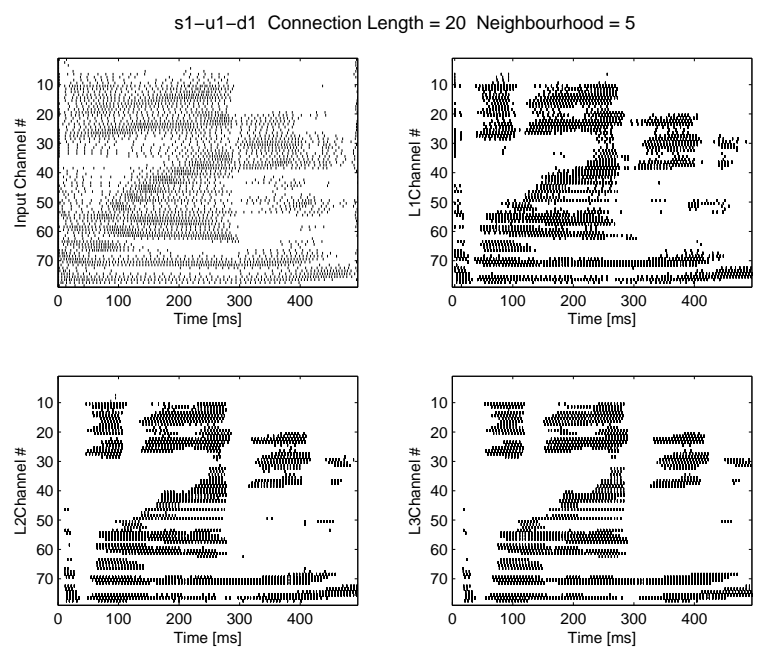

Figure 11: Neighbourhood Radius $=5$, Connection Length $=20$ for Sample: s1-u1-d1. Single and low frequency 'noisy' spikes are removed with each successive layer of this connectivity regime.

As can be seen from the figure the small neighbourhood radius, and slightly larger connection length do not result in a synchronous state. Instead, the connectivity has served to remove noisy spikes and extract a clearer representation of the sound signal by sharpening the main contours of the spike distribution. This kind of noise reduction and edge enhancement capability has been observed in retina and cochlear nucleus cells, likely with similar regimes of connectivity. Figure 12 shows the subsequent mutual information over time calculation, as with the synchronous topology the mutual information peaks around $t=0$. The amount of mutual information is higher than for the synchronous networks (peaking at 0.93 bits).

The increase in information transmission is small in this first layer but subsequent layers need to be examined to see if the differences in information preservation are more pronounced. Thus the mutual information between the cochleagram and the consecutive layers of synchrony (max connection length), and the mutual information between the cochleagram and the successive neighbourhood layers (noise reduction topology with connection length $=20$; neighbourhood radius $=15$ ) are compared. Figure 13 shows the change in mutual information for the two topologies through successive layers, note that layer 0 refers to the mutual information

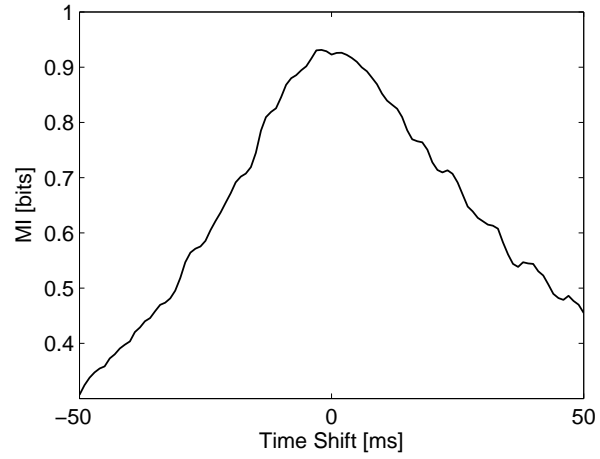

Figure 12: Mutual information between cochleagram and layer 1 output of topology with Neighbourhood Radius $=5$, Connection Length $=20$ with multiple time shifts for sample: s1-u1-d1

between the cochleagram and the encoded spikes.

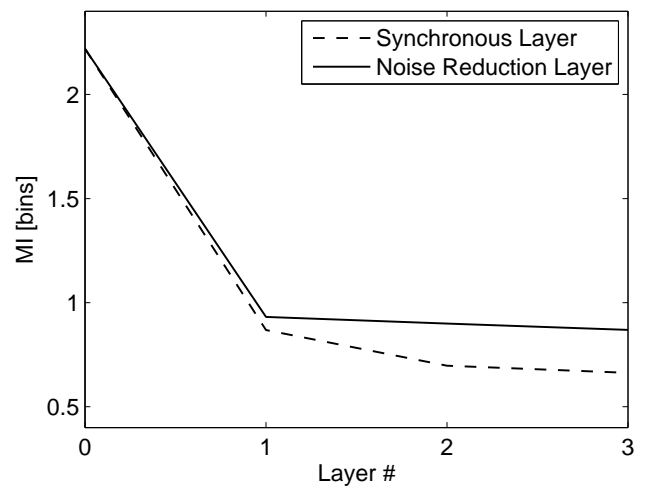

Figure 13: Mutual information between cochleagram and synchronous layers, and cochleagram and noise reduction layers for sample: s1-u1-d1

As can be seen from Figure 13, both the topologies lose some information from the encoding to the output of the first layer. However, what is interesting is what occurs in the subsequent layers, the noise reduction topology is more adept at preserving the information between layers, whilst the synchronous topology progressively loses information.

\section{Discussion}

A potential criticism for using the naive approach to the calculation of the mutual information arises with the calculation of mutual information between the cochleagram and the outputs spike rasers of the various laterally connected inhibitory layers. Since there is substantial connectivity between layers, in theory mutual information can be calculated across all frequency channels 
simulataneously. Unfortunately, calculating mutual information across 78 dimensions is computationally an intractable proposition at this time, particularly because of the binary word lengths and resulting amounts of data such a calculation would require. Furthermore, in this work it was desirable to determine the relationship between the input channels and the corresponding output channels, for other applications involving laterally connected neurons this may not be of interest. Future work will investigate how the methodology presented here can be extended to this multi-information case. For the time being the authors are satisfied that the clear tonotopic organisation between the layers is sufficient to capture the mutual information if only in relative terms (relative between the encoding and the various output layers).

\section{Conclusions}

The work presented is aimed at providing insight into the effects of modifying lateral inhibitory connectivity on a layer of LIF spiking neurons [17]. In contrast to work in reservoir computing where a typically random conectivity ethos is preferred, this paper represents ongoing work on attempting to quantify what the effects of particular lateral connectivity regimes are on neural coding and information preservation. In this way, two connectivity parameters were defined that modify the connectivity in a conceptually meaningful way. The connection length parameter dictates how far away (tonotopically) lateral neurons are connected to one another. Conversely, the neighbourhood radius parameter dictates how tonotopically distant neurons can be that are not connected laterally. The interplay between the two parameters is the main focus of this paper. Initially experiments consider modifying the connection length parameter alone, in topologies where spiking neurons are sparsely laterally connected to each other with inhibitory synapses, and investigate to what extent synchrony is affected by increasing the degree of lateral connectivity (increasing connection length). In agreement with other research it is discovered that synchrony results from sparse connectivity [4]. However, as connectivity increases synchrony becomes more defined. Similarly multiple layers of synchrony further reduces spike output and produces more pronounced synchronous states.

In later experiments the neighbourhood radius parameter and its interplay with inhibitory connectivity regimes (as dictated by the connection length parameter) are investigated. Most synchrony experiments advocate an all-things-being-equal approach where synaptic weights for example are kept the same in order to facilitate synchrony (and also because of a lack of a rationale for varying weights). In experiments where weights are modified [6] activity is shown to unsynchronise. Similarly, adding a neighbourhood radius parameter which dictates a local lack of connectivity for neurons results in spikes that are not synchronised. However, with large connection lengths, neighbourhoods of neurons compete with one another, with 'winning' neighbourhoods suppressing all others. Interestingly, when the neighbourhood radius is small and the connection length parameter is only slightly larger, the connectivity serves to reduce noisy spikes whilst preserving edges in the spike rasters. Such connectivity experiments could shed some light on similar capabilities of retina cells and cells in the cochlear nucleus, which are known to extensively employ lateral inhibition. In summary, this work illustrates some interesting findings with experiments with various forms of spiking neuron connectivity, and in particular seeks to unravel some of the functionality of inhibitory neurons. Spiking neural network topologies that implement this kind of connectivity could have some significantly improved capabilities for pattern recognition problems.

The work presented augments previously published work [10] by introducing information theoretic analysis to quantify the mutual information between the cochleagram stimulus, spiker encoding algorithm, synchronous, and noise reduction topologies. The analysis shows the effectiveness of the encoding and uses this as a relative baseline for comparing the information lost by the synchronous layer (maximum connection length) to the information lost by the combination of neighbourhood radius and connection length (noise reduction) topology. The neural processing in all of the networks presented show a large reduction in mutual information once the encoded spikes are processed by the various lateral inhibitory networks. This is to be expected as it is the networks task to transform the input encoding to something more useful. In the case of the synchronous networks this reduction in mutual information is more aggressive, verified by the accompanying raster plots which show periodic removal of spikes. Contrastingly, the noise reduction networks show a preservation of mutual information through the various layers, again as can be seen by the accompanying rasters that show that although noisy spikes have been removed, the remaining spikes still qualitatively represent the stimulus accurately. Additionally, the total maximum information for the synchronous and noise reduction topologies demonstrate that where the former topologies progressively lose information about the stimulus, the latter re- 
tain it. This clearly points to the detrimental effects of such epileptic synchrony in information terms (albeit with a naive mutual information approach). Therefore clearly demonstrating that near-sycnronous states (such as those employed by the noise reduction networks) are far more useful for neural processing than full synchronous states.

\section{Acknowledgements}

The authors wish to thank Dr. Daniel Polani, Christoph Salge, Malte Harder, and all the members of the Sepia group of the Adaptive Systems Research Group, School of Computer Science, University of Hertfordshire, for their many useful comments and insight into the information theoretic concepts discussed throughout the paper.

This research is supported under the Centre of Excellence in Intelligent Systems (CoEIS) project, funded by the Northern Ireland Integrated Development Fund and InvestNI.

\section{References}

[1] C. von der Malsburg, and W. Schneider, "A neural cocktail-party processor," Biological Cybernetics, vol. 54, pp. 29-40,1986.

[2] C. M. Gray, P. Knig, A. K. Engel, and W. Singer, "Oscillatory responses in cat visual cortex exhibit inter-columnar synchronization which reflects global stimulus properties," Nature, vol. 338, no. 6213, pp. 334-337, 1989.

[3] C. Van Vreeswijk, L. F. Abbott, and G. B. Ermentrout, "When inhibition not excitation synchronizes neural firing," Journal of Computational Neuroscience, vol. 1, no. 4, pp. 313-321, 1994.

[4] C. Börgers, and N. Kopell, "Synchronization in networks of excitatory and inhibitory neurons with sparse, random connectivity," Neural Computation, vol. 15, no. 3, pp. 509-538, 2003.

[5] M. Dhamala, V. Jirsa, and M. Ding, "Enhancement of neural synchrony by time delay," Physical Review Letters, vol. 92, no. 7, pp. 074104-1-074104-6, 2004.

[6] E. V. Lubenov, and A. G. Siapas, "Decoupling through synchrony in neuronal circuits with propagation delays," Neuron, vol. 58, no. 1, pp. 118-131, 2008.

[7] F. Ratliff, H. K. Hartline, and W. H. Miller, "Spatial and temporal aspects of retinal inhibitory interaction," Journal of the Optical Society of America, vol. 53, no. 1, pp. 110-120, 1963.

[8] S. Shamma, "On the role of space and time in auditory processing," Trends in Cognitive Sciences, vol. 5, no. 8, pp. 340-348, 2001.

[9] R. G. Lyon, "A computational model of filtering, detection, and compression in the cochlea," Proc. IEEE International Conference on ICASSP'82, vol. 7, 1982.

[10] C. Glackin, L. Maguire, L. McDaid, and J. Wade, "Lateral Inhibitory Networks: Synchrony, Edge Enhancement, and Noise Reduction," Proc. IEEE International Joint Conference on Neural Networks (IJCNN), 2011.

[11] R. Linsker, "Self-organisation in a perceptual network," IEEE Computer, vol. 21, pp. 105.117, 1988.

[12] L. Paninski, "Estimation of entropy and mutual information," Neural Computation, vol. 15, no. 6, pp. 1191-1253, 2003.
[13] R. R. de Ruyter van Steveninck, G. D. Lewen, S. P. Strong, R. Koberle, and W. Bialek, "Reproducibility and variability in neural spike trains," Science, vol. 275, no. 5307, pp. 1805-1808, 1997.

[14] M. Liberman. (1993). TI46 speech corpus, (Linguistic Data Consortium)[Online]. Available: http: //www. Idc. upenn. edu/Catalog/CatalogEntry. jsp?catalog Id=LDC93S9

[15] L. C. W. Pols, "Spectral analysis and identification of Dutch vowels in monosyllabic words," Ph.D. dissertation, Free University, Amsterdam, The Netherlands, 1966.

[16] M. Slaney. (1998) Auditory toolbox: A MATLAB Toolbox for auditory modeling work, (Interval Research Corporation).[Online]. Available: http://web.interval.com/ papers/1998-010.

[17] W. Gerstner and W. M. Kistler, "Spiking Neuron Models: Single Neurons, Populations, Plasticity," Cambridge University Press, 2002.

[18] H. de Garis, N. E. Nawa, M. Hough, and M. Korkin, "Evolving an Optimal De/Convolution Function for the Neural Net Modules of ATRs Artificial Brain Project," Proc. International Joint Conference on Neural Networks, IJCNN'99, vol. 1, pp. 438443, 1999.

[19] B. Schrauwen and J. Van Campenhout, "BSA, a fast and accurate spike train encoding scheme," Proc. International Joint Conference on Neural Networks, vol. 4, pp. 2825-2830, 2003.

[20] S. A. Wills. (2001) Recognising speech with biologicallyplausible processors, (Hamilton Prize) [Online]. Available: http://www.inference.phy.cam.ac.uk/saw27/ hamilton.pdf.

[21] L. S. Smith, "Robust sound onset detection using leaky integrate-and-fire neurons with depressing synapses," IEEE Transaction on Neural Networks, vol. 15, no. 5, pp. 1125-1134, 2004.

[22] C. Glackin, L. Maguire, and L. McDaid, "Feature Extraction from Spectro-temporal Signals using Dynamic Synapses, Recurrency, and Lateral Inhibition," Proc. IEEE World Congress Computational Intelligence (WCCI), pp. 1-6, 2010.

[23] T. M. Cover, and J. A. Thomas, "Elements of information theory," John Wiley \& Sons, 1991.

[24] L. Olsson, C. L. Nehaniv, and D. Polani, "Sensor adaptation and development in robots by entropy maximization of sensory data," Proc. Computational Intelligence in Robotics and Automation (CIRA), pp. 587-592, 2005.

[25] J. P. Nadal, and N. Parga, "Sensory coding: information maximization and redundancy reduction," Neural Information Processing, vol. 7, no. 1A-2, pp. 164-171, 1999.

[26] J. J. Hopfield and C. D. Brody, "What is a moment? Transient synchrony as a collective mechanism for spatiotemporal integration," Proc. National Academy of Sciences, vol. 98, no. 3, pp. 1282-1287, 2001.

[27] L. F. Abbott, "The timing game," Nature Neuroscience, vol. 4, no. 2, pp. 115-116, 2001. 